\title{
Evaluación del ajuste entre las competencias académicas y las profesionales de los titulados del grado de Diseño Gráfico: hacia un dispositivo de diagnóstico
}

\author{
Lluc Massaguer Busqueta \\ EINA. Centre Universitari de Disseny i Art de Barcelona. España. \\ mmassaguer@eina.cat \\ José Tejada Fernández \\ Universitat Autònoma de Barcelona. España. \\ jose.tejada@uab.cat
}

Recibido: 22/12/2019

Aceptado: $14 / 4 / 2020$

Publicado: 29/1/2021

\section{Resumen}

El análisis del ajuste entre los estudios realizados y la ocupación del egresado permite conocer hasta qué punto el ámbito profesional demanda las competencias alcanzadas. En este marco nos planteamos como objetivo verificar la correspondencia entre las competencias del plan de estudios de Diseño Gráfico y la demanda competencial de la profesión. La investigación ha seguido básicamente el método Delphi modificado y ha realizado dos rondas de aplicación sucesivas. La muestra la constituyen egresados $(n=26)$, profesionales empleadores $(n=19)$ y académicos $(n=19)$, siendo el cuestionario y el focus group los instrumentos diseñados y validados ad hoc que integran las competencias transversales y específicas del perfil profesional. Las conclusiones apuntan a un alto consenso, en particular entre egresados y empleadores, respecto de qué competencias son necesarias para la formación de los diseñadores. Además, se ha podido verificar la necesidad de establecer un dispositivo de diagnóstico para ajustar las competencias académicas a las competencias profesionales.

Palabras clave: competencias académicas; competencias profesionales; diseño gráfico; egresados; educación universitaria

Resum. Avaluació de l'ajustament entre les competències acadèmiques i les professionals dels titulats del grau de Disseny Gràfic: cap a un dispositiu de diagnòstic

L'anàlisi de l'ajust entre els estudis realitzats i l'ocupació del diplomat permet conèixer fins a quin punt l'àmbit professional demana les competències assolides. En aquest marc ens plantegem com a objectiu verificar la correspondència entre les competències del pla d'estudis de Disseny Gràfic i les necessitats competencials de la professió. La investigació ha seguit bàsicament el mètode Delphi modificat i ha realitzat dues rondes d'aplicació successives. La mostra la constitueixen diplomats $(n=26)$, professionals ocupadors $(n=19)$ i acadèmics $(n=19)$. El qüestionari i la reunió de grup són els instruments dissenyats $\mathrm{i}$ validats ad hoc, a més, també ha integrat les competències transversals i específiques del 
perfil professional. Les conclusions apunten a un alt consens, en particular entre titulats i ocupadors, respecte de quines competències són necessàries per formar els dissenyadors. A més, s'ha pogut verificar la necessitat d'establir un dispositiu de diagnòstic per ajustar les competències acadèmiques a les competències professionals.

Paraules clau: formació de l'emprenedoria; alerta emprenedora; factors d'emprenedoria; capacitats emprenedores

Abstract. Evaluation of the match between academic and professional competences of graphic design graduates: Towards a diagnostic device

The analysis of the match between study programmes and graduates' occupation enables determining to what extent the professional field demands the competences acquired. Within this framework, this study aims to verify the correspondence between the competences of the bachelor's degree in graphic design and the competences required in the profession. To conduct the research, the modified Delphi method was used. Two rounds were implemented successively. The sample consisted of graduates $(n=26)$, professionals-employers $(n=19)$ and academicians $(n=19)$. Questionnaires and a focus group were the instruments employed, which integrated transversal and specific competences of the professional profile. The instruments were validated ad hoc. The conclusions point to a high consensus, in particular among graduates and employers, regarding which competences are necessary for the training of designers. In addition, the research showed the need for a diagnostic device to achieve a better fit between academic competences and professional ones.

Keywords: academic competences; professional competences; graphic design; graduates; higher education

\author{
Sumario \\ 1. Introducción 4. Discusión y conclusiones \\ 2. Metodología Referencias bibliográficas \\ 3. Resultados
}

\title{
1. Introducción
}

La entrada de la educación superior al EEES (Espacio Europeo de Educación Superior) causó un impacto considerable en la manera de entender el proceso de enseñanza-aprendizaje. Se pasó de una universidad focalizada en la lógica de la enseñanza y el aprendizaje de contenidos disciplinarios a una universidad en la que resulta fundamental el logro y el desarrollo de competencias que permitan, a quien se gradúa, desarrollarse satisfactoriamente en un entorno social y económico cambiante, incluso innovando en sus respectivos ámbitos de trabajo.

Este cambio paradigmático pone a la competencia profesional como referente fundamental de la formación, de manera que afecta tanto al diseño, a los objetivos y a los contenidos, a los métodos y a la evaluación como a aspec- 
tos cruciales como la transformación de las actividades educativas o la consolidación de una sociedad en red que modifica la interacción educativa entre profesorado y estudiantes, hasta tal punto que han cambiado las formas de enseñar y de aprender (Vega, 2017).

El enfoque que se da a este estudio es la aceptación de que la naturaleza de las competencias puede permitir conocer y entender los fundamentos en que se basa el perfil profesional de un empleo, así como el de una titulación universitaria. La definición de un perfil de competencias para una titulación se ve ligado a la realidad en la que se encuentra, al contexto socioeconómico-laboral, al tipo de conocimiento que supone y a los valores que engendra esta relación. Es por ello que se valoran todos estos aspectos como parte importante de su ejercicio.

El análisis de las relaciones entre el ámbito formativo y el profesional tiene trascendencia a la hora de proporcionar información objetiva que sirva como base para la toma de decisiones por parte de los agentes educadores, pero también hay que tener en cuenta que la adecuación de las decisiones adoptadas tendrá un efecto más allá del ámbito académico, lo que trascenderá a la sociedad y a su dinámica económica.

Analizar el ajuste entre los estudios realizados y la ocupación de quien se gradúa permite conocer hasta qué punto el ámbito profesional demanda las competencias alcanzadas por los titulados mediante la formación universitaria (Bartual y Turmo, 2016; Cabrera, López López y Portillo, 2016; Freire, Teijeiro y Pais, 2013; OECD, 2015; Rodríguez-Esteban, Vidal y Vieira, 2019; Somers, Cabus, Groot y Maassen van den Brink, 2016; Verhaest, Sellami y Van der Velden, 2017).

Según Michavila, Martínez, Martín-González, García-Peñalvo y CruzBenito (2016), cualquier desajuste puede explicarse, al menos, por dos causas no necesariamente excluyentes. Puede tratarse de una inadecuación de los estudios realizados, por lo que se debería plantear una reorientación de la formación, o bien de una estructura productiva profesional que no es capaz de aprovechar los recursos disponibles o lo hace de forma insuficiente, lo que también podría llevar a considerar actuaciones para su mejora (Centro Europeo para el Desarrollo de la Formación Profesional, CEDEFOP, 2014).

Queremos señalar que existen investigaciones recientes realizadas en otros países europeos (CEDEFOP, 2014, 2016, 2017; Iriondo y Pérez Amaral, 2016; Kucel y Vilalta, 2013; Morgado, Sequeira, Santos, Ferreira-Lopes y Reis, 2015; Pizarro-Barceló y Herrero-Montagud, 2016; Planas, 2014; Teichler, 2015), camino al que se suma la presente investigación, que sugieren que la no correspondencia entre formación y empleo no constituye necesariamente, ni en todos los casos, un desajuste negativo y que el fenómeno de la «inadecuación» entre especialidades probablemente tenderá a crecer, pero sus efectos negativos disminuirán. Así lo indican las nuevas evoluciones experimentadas, tanto en los itinerarios formativos de los jóvenes como en el contenido de los puestos de trabajo (Consejo Económico y Social, CES, 2015; Planas, 2014; Teichler, 2015). 
En el contexto actual los ámbitos productivo y educativo caminan a diferentes velocidades. Generalmente, los procesos formativos necesitan de largos trámites para modificar sus planes de estudio, a menudo por motivos políticos y/o burocráticos, lo que requiere más tiempo de lo que el sector profesional está dispuesto a esperar cuando solicita resolver unas necesidades rápidamente cambiantes, reflejadas en un determinado perfil de cualificaciones. Es aquí donde nace la brecha entre oferta y demanda de competencias. La tecnología y los procesos de producción se ajustan a una velocidad mayor que a la que se revisan, se rediseñan y se ejecutan los planes de estudio (Agencia para la Calidad del Sistema Universitario de Cataluña, AQU, 2017; CIDAC, 2014; Del Vecchio, 2014; Lluch, Fernández-Ferrer, Pons y Cano, 2017).

Con el fin de concretar en qué tipo de profesional nos centramos, intentamos partir de la actividad del diseñador gráfico refiriéndonos a su perfil "clásico", a aquel profesional que desarrolla un proyecto de modo conceptual y/o formal. La línea que antes delimitaba de manera bastante definida los perfiles de diseñador ahora está más diluida. Las competencias profesionales de quien se dedica al diseño gráfico han sido valoradas por diferentes investigadores y profesionales (Folch, 2016; González-Mardones; 2016; Vega, 2017). Además, nosotros incluimos en la revisión las establecidas en el libro blanco de títulos de grado en Bellas Artes, Diseño y Restauración de la Agencia Nacional de Evaluación de la Calidad y Acreditación, ANECA (2004).

En este marco nos planteamos el objetivo en nuestra investigación: analizar la correspondencia entre las competencias en que se basa el plan de estudios de Diseño Gráfico y la demanda que hace la profesión, de cara a la elaboración de un sistema diagnóstico que se pueda aplicar en diferentes momentos y de manera periódica, para identificar el nivel de ajuste entre las competencias académicas impartidas en la universidad y las competencias profesionales requeridas en el ámbito sociolaboral.

\section{Metodología}

\subsection{Diseño}

Se enfoca el estudio desde un paradigma interpretativo a través de un método mixto secuencial, donde el método cuantitativo ha precedido a la utilización de un método cualitativo, entrando en las especificidades de los datos para descubrir las categorías, las dimensiones y las interrelaciones (HernándezSampieri, Fernández-Collado y Baptista-Lucio, 2014).

Dada la problemática general del ajuste entre las competencias académicas y las profesionales, nos hemos basado en un diseño de investigación de estudio de caso, analizando el objeto de análisis en su contexto, considerándolo relevante tanto para comprobar, ilustrar o construir una teoría o una parte de ella, como por su valor intrínseco, puesto que puede ser transferida a otras coyunturas. En este análisis partimos de la clasificación de tipos de estudio de caso de Coller (2000), al caracterizarlo como caso único, no múltiple, ya que se 
centra en lo que acontece y cómo pasa en una sola institución, caso de estudio de objeto que se centra en el análisis de un fenómeno en una organización concreta; caso instrumental, en cuanto se ilustra una teoría sobre el funcionamiento de un fenómeno, y caso típico, ya que se considera a más de un grupo y reúne las características del mismo de dichos grupos; caso contemporáneo, dado que se centra en un fenómeno que acontece durante el propio desarrollo del estudio, y caso analítico, que estudia sin hipótesis previas y, a partir de las observaciones, ilustra la teoría y realiza proposiciones sobre el funcionamiento del fenómeno siguiendo el método inductivo de investigación.

El análisis se sitúa en el ámbito de los estudios universitarios de Diseño Gráfico a partir del caso de EINA, Centro Universitario de Diseño y Arte de Barcelona, adscrito a la Universidad Autónoma de Barcelona.

Figura 1. Diseño del estudio

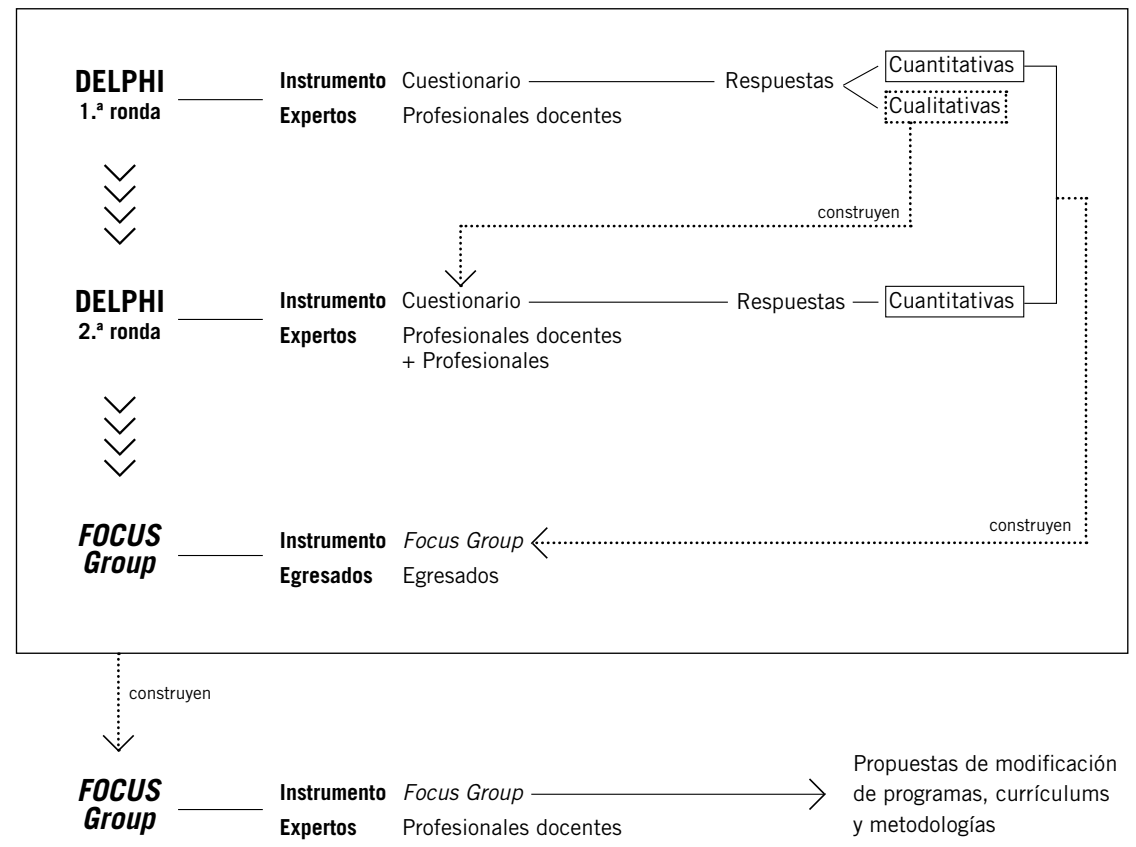

Fuente: elaboración propia.

La investigación ha seguido básicamente el método Delphi modificado (Massaguer, 2018; Vallor, Yates y Brody, 2016), con dos rondas de aplicación sucesivas a partir de la utilización sistemática del juicio experto: la primera a personas conocedoras del ámbito, tanto académico como profesional, todas ellas profesionales docentes; la segunda se ha basado en una ampliación de la muestra, lo que ha permitido concretar el conjunto de 
indicadores que construyen la herramienta de diagnóstico, parte del resultado de la investigación.

Aunque la mayoría de las características de este método se perciben como ventajas, desde nuestro estudio entendemos principalmente que los resultados son cuantitativa y cualitativamente valiosos, por estar facilitados por grupos profesionales especialmente conocedores del tema y del contexto real, operando con una muestra bastante relevante al efecto - en torno a veinte personas por grupo de referencia (García, Aquino, Guzmán y Medina, 2012; Cabero e Infante, 2014).

\subsection{Participantes}

La población la constituyen los recién egresados del nuevo grado de Diseño Gráfico de EINA, los docentes y directivos implicados en el estudio, así como profesionales empleadores.

En la primera ronda Delphi se ha consultado a 19 personas expertas del ámbito del diseño gráfico, tanto académico como profesional. En la segunda ronda Delphi se ha ampliado la muestra a 64 personas, añadiendo expertos profesionales empleadores en el ámbito del diseño gráfico $(n=19)$, pero que no forman parte del mundo académico, además de integrar a los egresados $(n=26)$. El coeficiente de competencia experta (Cabero y Barroso, 2013) ha sido de 0,88 para el primer grupo de expertos y de 0,73 para el segundo $\mathrm{K}=1 / 2(\mathrm{Kc}+\mathrm{Ka})$, entendiendo Kc como el coeficiente de conocimiento sobre el tema en formato de autoevaluación (de 0 a 10) multiplicado por 0,1, y Ka como el coeficiente de argumentación, obtenido a partir de los años de experiencia docente y profesional: 1 para más de 15 años, 0,8 entre 5 y 14 años, y 0,5 para menos de 5 años).

El primer focus group ha contado con la participación de egresados del grado de Diseño desde su primera implantación (8 egresados, 2 por cada promoción, desde el curso 2012-2013 hasta el curso 2015-2016). El segundo focus group ha sido formado por 6 personas que ocupan un cargo académico en el momento de realizar la investigación en la institución.

Tanto en las dos rondas Delphi como en los dos focus group la técnica de muestreo ha sido intencional, no probabilística. En el primer caso, el criterio utilizado ha sido la consideración del coeficiente de experticia, y en el segundo caso, una representación por promoción de egresados y académicos con cargo.

\subsection{Instrumentos}

Ante la necesidad de acceder a información, cuantitativa y cualitativa, mediante diversidad de informantes (empleadores, egresados y profesores), se ha considerado el uso de diferentes técnicas e instrumentos, combinando básicamente cuestionarios y focus group. Partimos del principio de la multivariedad y la triangulación instrumental, que nos lleva a integrar y conjugar coherentemente diferentes maneras de recoger evidencias de la competencia profesional (Tejada y Ruiz, 2016). 
El cuestionario de la primera ronda Delphi, diseñado ad hoc, teniendo como referencia a las competencias del título (memoria VERIFICA), fue sujeto a una fase piloto (aproximativa) en la que se verificó su funcionamiento. Se estructuró en 45 preguntas cerradas (ítems sobre competencias, 28 específicas y 17 transversales) y 5 ítems abiertos sobre el futuro inmediato del diseño gráfico, además de 8 preguntas iniciales para medir el coeficiente de competencia y experticia. En este caso, la valoración también es doble sobre las competencias del perfil: su importancia para el ejercicio profesional actual y su importancia para el futuro (escala de 1 —nada importante- a 4 -muy importante-).

El cuestionario de la segunda ronda Delphi está formado por 67 ítems, todos ellos cuantitativos, fruto de los resultados emergentes de la primera ronda. Como aconseja la metodología Delphi, los ítems objeto de tratamiento en la segunda ronda proceden de las preguntas abiertas formuladas en la primera ronda, se han categorizado y contrastado entre todos los expertos y se han organizado conceptualmente. Se han estructurado en cinco partes (datos profesionales, cambios, competencias específicas, competencias transversales y comentarios).

El punto inicial de los focus group es el de los resultados obtenidos en las dos rondas Delphi. Se han considerado 12 indicadores. Se parte de las competencias que surgieron en la segunda ronda valoradas como muy importantes y se analiza si dichas competencias fueron logradas en la formación, también se plantean las competencias que ganarán en importancia en los próximos 5 años, las que no figuran en el plan de estudios y los cambios contextuales probables.

\subsection{Procedimiento}

Para dotar la investigación de rigurosidad y credibilidad científica se han validado los instrumentos consultando a un grupo de jueces con experiencia en el campo de la metodología y la formación universitaria del ámbito del diseño. Se les pedía valorasen el cuestionario en cada uno de sus ítems con criterios de univocidad, pertinencia e importancia. Con el mismo objetivo, mediante el coeficiente Alpha de Cronbach, se ha verificado la consistencia interna de los cuestionarios, tanto de la primera ronda $(0,96 \mathrm{alpha})$ como de la segunda $(0,87)$.

La recogida de información se ha realizado en diversas etapas: correo electrónico a todos los egresados con especificación del cuestionario Google forms; primera ronda Delphi, con envío de correo electrónico a los expertos del cuestionario Google forms en febrero de 2016, y segunda ronda Delphi en junio de 2016, una vez evaluada la ronda anterior.

Con los datos obtenidos en la aplicación de las dos rondas Delphi se ha realizado una propuesta de profundización para tratar en dos focus group. Se ha elegido utilizar esta estrategia cualitativa de recogida de datos para promover la interacción grupal, puesto que ofrece información de primera mano, 
estimula la participación mediante un carácter flexible y abierto y presenta una alta validez subjetiva.

Para el análisis de la información obtenida mediante la técnica de los cuestionarios se ha utilizado el paquete estadístico SPSS (Statistical Package for Socials Sciences), versión 19, implicando tanto estrategias de análisis estadístico descriptivo como inferencial: correlaciones de Pearson y pruebas t-Student o análisis de varianza para comparar entre grupos. Asimismo, se ha utilizado el programa MaxQDA para el análisis cualitativo de la información obtenida mediante el focus group, considerando el análisis de contenido realizado, con la aplicación del sistema categorial utilizado en línea con los indicadores utilizados.

\section{Resultados}

Si nos adentramos en los resultados obtenidos en la aplicación del sistema diagnóstico creado en la definición del perfil competencial del diseñador gráfico, verificamos, en la primera ronda Delphi, la valoración de la importancia de las competencias actualmente presentes en el plan de estudios.

Como puede apreciarse en la tabla y en las gráficas siguientes, globalmente podemos considerar que todas ellas han sido valoradas entre «importantes» $\mathrm{y}$ «muy importantes» por el grupo de expertos académicos, con excepción de las competencias específicas CE16, CE17 y CE24, cuya importancia es media-baja.

Si comparamos las competencias actuales y futuras, destaca que, en el $85 \%$ de los casos, la valoración acerca de la importancia de las competencias específicas previstas para un futuro próximo es más alta que en la actualidad.

Ello es indicador del valor actual para el perfil académico de dichas competencias y también se verifica el incremento de importancia para muchas de ellas en el futuro.

Tabla 1. Competencias del perfil profesional del diseño gráfico según los expertos académicos

\begin{tabular}{|c|c|c|c|}
\hline \multirow[b]{2}{*}{ Competencias específicas } & \multicolumn{3}{|c|}{ 1. ${ }^{a}$ RONDA DELPHI } \\
\hline & Actual & Futuro & Sign. \\
\hline 1. Detección de problemas de diseño & 3,42 & 3,79 & 0,031 \\
\hline $\begin{array}{l}\text { 2. Aportación de soluciones alternativas a los problemas de diseño } \\
\text { detectados }\end{array}$ & 3,26 & 3,74 & 0,008 \\
\hline 3. Evaluación de la viabilidad de los proyectos de diseño & 3,53 & 3,89 & 0,031 \\
\hline $\begin{array}{l}\text { 4. Realización de un programa de usos y funciones para el desarrollo } \\
\text { de un proyecto de diseño }\end{array}$ & 3,21 & 3,37 & \\
\hline 5. Exposición y razonamiento oral de los proyectos de diseño & 3,37 & 3,58 & \\
\hline 6. Exposición y razonamiento escrito de los proyectos de diseño & 3,05 & 3,37 & \\
\hline 7. Interpretación de proyectos de diseño & 3,26 & 3,47 & 0,042 \\
\hline 8. Valoración crítica de proyectos de diseño & 3,32 & 3,53 & 0,042 \\
\hline $\begin{array}{l}\text { 9. Conocimiento de los procesos, las tecnologías y los costes de fabri- } \\
\text { cación y producción de los proyectos de diseño desarrollados }\end{array}$ & $-3,58$ & 3,79 & \\
\hline
\end{tabular}


10. Formalización expresiva a partir de los materiales y sus procesos $\begin{array}{llll}3,37 & 3,63 & 0,021\end{array}$ de transformación

11. Utilización de los recursos expresivos de la fotografía en función de $3,42 \quad 3,58$ las necesidades comunicativas del proyecto de diseño

12. Uso adecuado del dibujo

$3,05 \quad 3,16$

13. Uso adecuado del color

$3,58 \quad 3,74$

14. Uso adecuado de formatos, medidas y proporciones

$3,58 \quad 3,63$

15. Uso adecuado del volumen

$3,11 \quad 3,05$

16. Seguridad en el trazo y realización de bocetos rápidos

$2,68 \quad 2,63$

17. Medición y geometrización de espacios y objetos

$2,37 \quad 2,63 \quad 0,021$

18. Uso adecuado de las formas de representación habituales en el $\quad 3,37 \quad 3,47$ sector profesional del diseño al que va dirigido el proyecto

19. Elección de materiales y procesos de transformación que se adap- $3,37 \quad 3,68$ ten a las necesidades funcionales y expresivas de cada diseño

20. Uso adecuado de la tipografía y la maquetación de página $\quad 3,68 \quad 3,63$

21. Uso y dominio del software profesional adecuado para el proyecto $\begin{array}{lll}3,63 & 3,68\end{array}$ de diseño

22. Combinación adecuada de técnicas artísticas tradicionales con $3 \quad 3,05$ medios digitales de manipulación de la imagen

23. Edición de elementos audiovisuales con imágenes animadas y $\begin{array}{llll}2,84 & 3,58 & 0,000\end{array}$ sonido sincronizado

24. Conocimiento de instituciones y asociaciones que configuran el $\quad 2,42 \quad 2,37$ mundo profesional del diseño

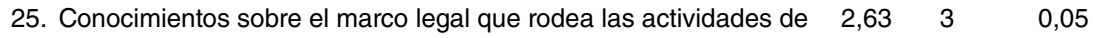
diseño

26. Uso intencionado de recursos gráficos para sintetizar y mejorar la $\begin{array}{lll}3,05 & 3,68 & 0,001\end{array}$ comunicación

27. Aplicación de criterios ergonómicos y parámetros antropométricos y 2,95 $\quad 3,26 \quad 0,030$ perceptivos de acuerdo con las características de uso del proyecto

28. Comprensión del entorno económico y empresarial con quien se $\begin{array}{rrrr}3,21 & 3,63 & 0,028\end{array}$ desarrolla el proyecto

\begin{tabular}{lccc}
\hline Competencias transversales & 3,26 & 3,89 & 0,001 \\
\hline $\begin{array}{l}\text { 1. Capacidad para trabajar en equipo } \\
\text { 2. Coordinación, dirección y liderazgo de equipos de trabajo interdisci- } 3,05\end{array}$ & 3,68 & 0,001 \\
plinarios en diferentes especialidades del diseño & & \\
$\begin{array}{l}\text { 3. Planificación, organización, gestión, administración y estudio de } \\
\text { viabilidad de proyectos de diseño }\end{array}$ & 3,21 & 3,53 & 0,030 \\
4. Elaboración de informes profesionales & 2,79 & 3,16 & 0,015 \\
\hline 5. Capacidad de entendimiento con los agentes que intervienen en el 3,37 & 3,63 & \\
desarrollo de un proyecto de diseño & 3,42 & 3,68 & \\
6. Capacidad de iniciativa y espíritu emprendedor & 3,63 & 3,79 & \\
7. Capacidad resolutiva y de toma de decisiones & 3,58 & 3,89 & 0,030 \\
\hline 8. Motivación por la calidad & 2,89 & 3,58 & 0,002 \\
\hline 9. Adaptación al entorno profesional nacional e internacional & 3,42 & 3,79 & 0,005 \\
\hline 10. Atención a los cambios que se van produciendo & 3,42 & 3,58 & \\
11. Uso de la creatividad en contextos y situaciones diferentes & & & \\
\hline
\end{tabular}


12. Orientación de los diseños a valores de respeto al medio ambiente $\begin{array}{llll}2,84 & 3,68 & 0,001\end{array}$ y la sostenibilidad

13. Valoración y fomento de la accesibilidad para diferentes grupos de $\begin{array}{llll}2,84 & 3,42 & 0,002\end{array}$ usuarios y receptores

14. Valoración y preservación del patrimonio cultural, artístico y pai- $\begin{array}{llll}2,74 & 3,16 & 0,005\end{array}$ sajístico

15. Valoración de los principios deontológicos propios de la profesión $\quad 2,89 \quad 3,26$

16. Conocimientos de los nuevos lenguajes y propuestas culturales $\begin{array}{llll}3,21 & 3,58 & 0,015\end{array}$

17. Formalización (disposición positiva a los valores estéticos y forma $\quad 3,42 \quad 3,68$ en el diseño)

Fuente: Massaguer (2018).

Si entramos en detalle, podemos percibir el incremento de su importancia en un futuro próximo y en la actualidad. En el caso de las competencias específicas se trata de aquellas que tienen que ver de manera muy directa con los cambios que está sufriendo el contexto socioeconómico que nos rodea. Son competencias relacionadas con el uso intencionado de recursos gráficos para sintetizar y mejorar la comunicación (CE26), la comprensión del entorno económico y empresarial con quien se desarrolla el proyecto (CE28), los conocimientos sobre el marco legal que rodea las actividades de diseño (CE25) o la aplicación de criterios ergonómicos y de parámetros antropométricos y perceptivos de acuerdo con las características de uso del proyecto (CE27).

$\mathrm{Si}$ nos centramos en las competencias transversales, siguiendo la terminología de González y Wagenaar (2006), las que prevén un aumento de importancia en el futuro son mayoritariamente sistémicas, como por ejemplo la orientación de los diseños a valores de respeto al medio ambiente y la sostenibilidad (CT12), la valoración y fomento de la accesibilidad para grupos de usuarios y receptores con diversidad funcional (CT13), la valoración y preservación del patrimonio cultural, artístico y paisajistico (CT14), la atención a los cambios que se

Gráfico 1. Competencias específicas del perfil profesional del diseño gráfico según los expertos académicos

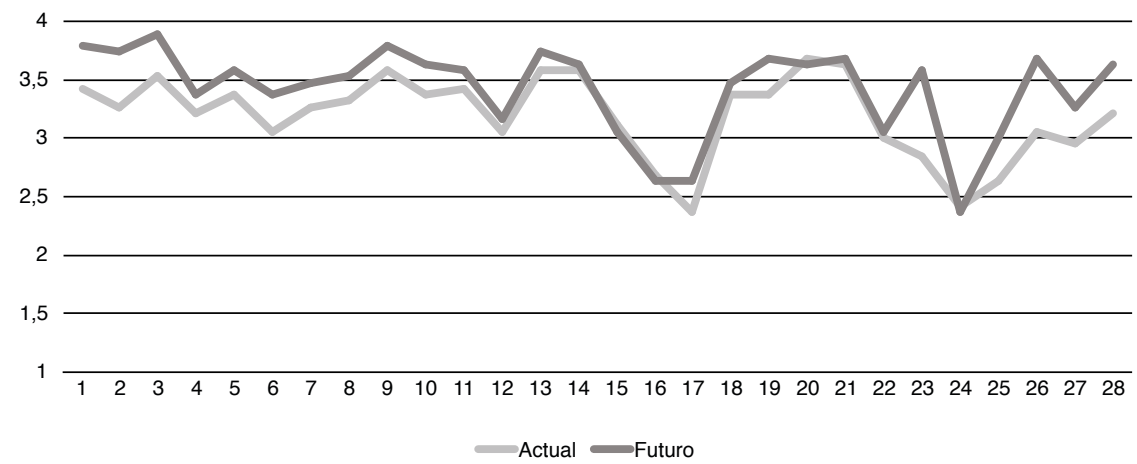

Fuente: Massaguer (2018). 
Gráfico 2. Competencias transversales del perfil profesional del diseño gráfico según los expertos académicos

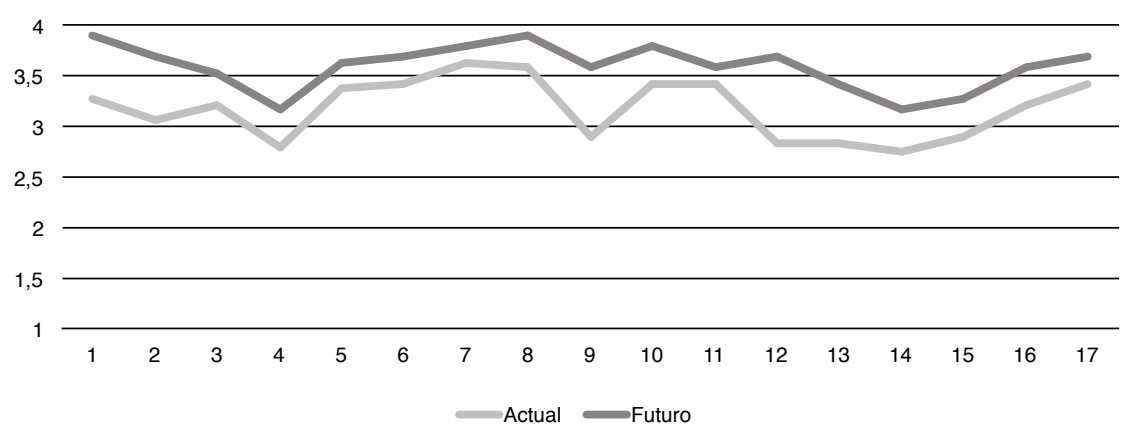

Fuente: Massaguer (2018).

van produciendo (CT10) y la adaptación al entorno profesional nacional e internacional (CT9). En las competencias interpersonales asociadas a la capacidad para trabajar en equipo (CT1) y a la coordinación, dirección y liderazgo de equipos de trabajo (CT2) igualmente se apunta un incremento significativo. Por contra, en las competencias instrumentales es donde se prevé menos aumento de importancia.

Se han categorizado las respuestas cualitativas a las preguntas abiertas en este primer cuestionario sobre nuevas competencias, descartando aquellas que equivalen a competencias contempladas en el actual plan de estudios de diseño y que dan pie a su inclusión en el cuestionario de la segunda ronda Delphi.

La síntesis de la aparición y del aumento de importancia de nuevas competencias, específicas y transversales, apunta en la siguiente dirección: entre las primeras cabe destacar las relacionadas con el conocimiento de programación en tecnologias digitales, la capacidad de trabajo en interfaz y usabilidad del usuario o teniendo en cuenta la interactividad del usuario en los proyectos de diseño, el dominio del uso de la tipografía para los nuevos formatos de publicación o la capacidad para entender la convivencia y el diálogo entre el soporte impreso y el digital y tener criterio para escogerlos según las necesidades del proyecto, entre otras. En cuanto a las transversales, podemos encontrar el uso fluido de diferentes idiomas, la gestión de redes de trabajo a distancia o la capacidad para comprender e interpretar otros modos de vivir (culturas, formas de pensar, religiones...).

La segunda ronda Delphi ha permitido valorar la importancia que tendrán las competencias construidas en la primera ronda en un futuro próximo (dentro de 5 años). En la tabla siguiente se presenta la comparativa entre las valoraciones, donde podemos destacar que las diferencias significativas solo se dan en tres de ellas. Todo ello es indicador de partida de una valoración bastante similar por parte de los agentes implicados en esta segunda ronda.

Globalmente cabe apuntar que la importancia atribuida al conjunto de competencias está en una valoración entre «importante» y «muy importante», 
si exceptuamos las competencias capacidad para trabajar en experiencias inmersivas interactivas (CE7), conocimiento de las tecnologias relacionadas con el tratamiento del color (CE13), capacidad de tratamiento y representación de gran volumen de datos (CE23) y capacidad de dominar el lenguaje visual más que el textual (CE24).

Tabla 2. Valoración de la importancia de las competencias profesionales en los próximos 5 años, según implicados

\begin{tabular}{|c|c|c|c|c|}
\hline Competencias específicas & Académico & Egresado & $\begin{array}{l}\text { Profesional } \\
\text { empleador }\end{array}$ & Sign. \\
\hline $\begin{array}{l}\text { 1. Conocimiento de programación en tecnolo- } \\
\text { gías digitales }\end{array}$ & 3,21 & 3,35 & 3,42 & \\
\hline 2. Capacidad de trabajo en UI/UX & 3,42 & 3,58 & 3,37 & \\
\hline $\begin{array}{l}\text { 3. Conocimiento de posicionamiento web } \\
\text { (SEO) }\end{array}$ & 2,84 & 3,15 & 2,95 & \\
\hline $\begin{array}{l}\text { 4. Dominio técnico de medios de comunicación } \\
\text { digitales }\end{array}$ & 3,37 & 3,15 & 3,05 & \\
\hline $\begin{array}{l}\text { 5. Dominio técnico para la creación de elemen- } \\
\text { tos relacionados con los entornos tridimen- } \\
\text { sionales }\end{array}$ & 2,95 & 2,92 & 2,74 & \\
\hline $\begin{array}{l}\text { 6. Capacidad para trabajar considerando la } \\
\text { interactividad del usuario en los proyectos } \\
\text { de diseño }\end{array}$ & 3,53 & 3,73 & 3,42 & \\
\hline $\begin{array}{l}\text { 7. Capacidad para trabajar en experiencias } \\
\text { inmersivas interactivas }\end{array}$ & 2,74 & 3,12 & 2,63 & \\
\hline $\begin{array}{l}\text { 8. Dominio de los criterios de usabilidad y } \\
\text { tenerlos en cuenta a la hora de desarrollar } \\
\text { proyectos de diseño }\end{array}$ & 3,58 & 3,77 & 3,58 & \\
\hline $\begin{array}{l}\text { 9. Capacidad para diseñar nuevos dispositivos } \\
\text { (tecnologías ponibles, consolas...) }\end{array}$ & 3,32 & 3,38 & 3,11 & \\
\hline $\begin{array}{l}\text { 10. Capacidad para formalizar en el ámbito del } \\
\text { diseño online }\end{array}$ & 3,47 & 3,65 & 3,47 & \\
\hline $\begin{array}{l}\text { 11. Comprensión del contenido de un proyecto } \\
\text { de diseño digital, considerando que su canal } \\
\text { de salida final puede variar en el futuro }\end{array}$ & 3,26 & 3,50 & 3,37 & \\
\hline $\begin{array}{l}\text { 12. Capacidad de realización de proyectos en } \\
\text { nuevos formatos digitales de publicación }\end{array}$ & 3,21 & 3,73 & 3,53 & 0,025 \\
\hline $\begin{array}{l}\text { 13. Conocimiento de las tecnologías relaciona- } \\
\text { das con el tratamiento del color }\end{array}$ & 2,63 & 3,12 & 3,00 & \\
\hline $\begin{array}{l}\text { 14. Dominio del uso de la tipografía para los } \\
\text { nuevos formatos de publicación }\end{array}$ & 3,42 & 3,62 & 3,26 & \\
\hline $\begin{array}{l}\text { 15. Capacidad para entender la convivencia y el } \\
\text { diálogo entre el soporte impreso y el digital y } \\
\text { criterio para escogerlos según las necesida- } \\
\text { des del proyecto }\end{array}$ & 3,63 & 3,42 & 3,63 & \\
\hline 16. Capacidad para diseñar diferentes formatos & 3,63 & 3,65 & 3,68 & \\
\hline $\begin{array}{l}\text { 17. Dominio de las técnicas y de los procesos } \\
\text { para la creación del nombre de una marca }\end{array}$ & 2,84 & 3,31 & 3,16 & \\
\hline 18. Dominio de estrategias de marketing & 2,95 & 3,38 & 3,11 & \\
\hline
\end{tabular}


19. Criterio para reconocer y escoger el contexto donde hay que actuar a través del diseño

\begin{tabular}{llll}
\hline 3,53 & 3,58 & 3,63 & \\
\hline 2,89 & 3,00 & 2,79 & \\
\hline 3,21 & 3,46 & 3,42 & \\
3,26 & 3,19 & 3,26 & \\
\hline 3,37 & 2,96 & 2,68 & 0,017 \\
3,11 & 3,19 & 2,74 & 0,050 \\
\hline 3,42 & 3,50 & 3,26 & \\
\hline 3,42 & 3,42 & 3,47 & \\
\hline
\end{tabular}

20. Uso adecuado (según los requerimientos del proyecto) de la ilustración

21. Capacidad de contextualizar los proyectos de diseño en el entorno cultural

22. Capacidad para crear nuevos lenguajes visuales

23. Capacidad de tratamiento y representación de gran volumen de datos

24. Capacidad de dominar el lenguaje visual más que el textual

25. Dominio de las capacidades propias de un project management

26. Uso de la investigación en los proyectos de diseño

\section{Competencias transversales}

1. Uso fluido de diferentes idiomas

3,42

3,54

3,42

2. Conocimientos de organización empresarial

2,84

2,92

3,11

3. Gestión de redes de trabajo a distancia

3,53

3,31

4. Conocimientos en tareas contables

2,68

2,50

2,63

5. Gestión en la integración de profesionales

3,53

3,31

3,53
de diferentes ámbitos en el proyecto de diseño

6. Conocimiento técnico en diversas disciplinas, dentro o fuera del diseño

7. Conocimientos en el ámbito comercial de gestión y trato con los clientes

8. Utilización de técnicas de comunicación

9. Capacidad de colaboración con diferentes agentes

10. Capacidad de trabajo en proyectos participativos y/o ecosistémicos

11. Comprensión del papel del diseñador como agente para el cambio social

12. Motivación para el reciclaje y la actualización constante de conocimientos tecnológicos

13. Capacidad para comprender e interpretar otros modos de vivir (culturas, formas de pensar, religiones...)

14. Conocimientos en el ámbito de la psicología social

$\begin{array}{lll}3,32 & 3,12 & 3,21\end{array}$

$\begin{array}{lll}3,26 & 3,38 & 3,42\end{array}$

$\begin{array}{lll}3,53 & 3,38 & 3,47\end{array}$

$\begin{array}{lll}3,74 & 3,46 & 3,58\end{array}$

$\begin{array}{lll}3,47 & 3,31 & 3,37\end{array}$

$\begin{array}{lll}3,16 & 3,19 & 3,32\end{array}$

$\begin{array}{lll}3,42 & 3,42 & 3,42\end{array}$

$\begin{array}{lll}3,47 & 3,54 & 3,47\end{array}$

$3,11 \quad 3,15 \quad 3,26$

15. Conocimientos en el ámbito de las humanidades como la filosofía o la ética

\begin{tabular}{lll}
3,16 & 2,96 & 3,16 \\
\hline 3,26 & 3,08 & 3,32
\end{tabular}

16. Conocimiento de las características generales de los principales métodos y tendencias de la historia del arte y del diseño

Fuente: Massaguer (2018). 
Si reparamos en las diferencias de valoración, en primer lugar, los expertos académicos creen que la capacidad de tratamiento y representación de gran volumen de datos (C23) tiene más importancia que la que manifiestan los egresados, y estos segundos, más que los expertos profesionales. Aunque esta diferencia es significativa estadísticamente hablando, dicha competencia es valorada como «bastante importante» por los tres tipos de perfil profesional.

En segundo lugar, la capacidad de realización de proyectos en nuevos formatos digitales de publicación (CE12) es valorada como "muy importante» por parte de los expertos egresados y profesionales, pero como «bastante importante» por parte de los expertos académicos.

Por último, la competencia relacionada con la capacidad de dominar el lenguaje visual más que el textual (CE24) es valorada por el grupo experto como bastante importante, aunque entre sus respuestas hay una diferencia estadísticamente significativa, con una evaluación más alta por parte de los egresados, una segunda puntuación por parte de los expertos académicos y una inferior por parte de los expertos profesionales.

Podemos concluir la lectura de resultados de la segunda ronda Delphi diciendo que el consenso en las respuestas es elevado y que, salvo las diferencias significativas encontradas, se percibe un acuerdo de opinión entre los diversos puntos tratados y los diferentes expertos.

La graduación que hace el grupo de expertos de la importancia de las competencias específicas en los próximos 5 años contienen como muy relevantes las competencias relacionadas con la usabilidad, la interactividad del usuario, la formalización en el ámbito del diseño online, la capacidad para diseñar diferentes formatos, el criterio para reconocer y escoger el contexto donde hay que actuar a través del diseño, la capacidad para entender la convivencialdiálogo entre

Gráfico 3. Valoración de la importancia de las competencias específicas en los próximos 5 años, según implicados

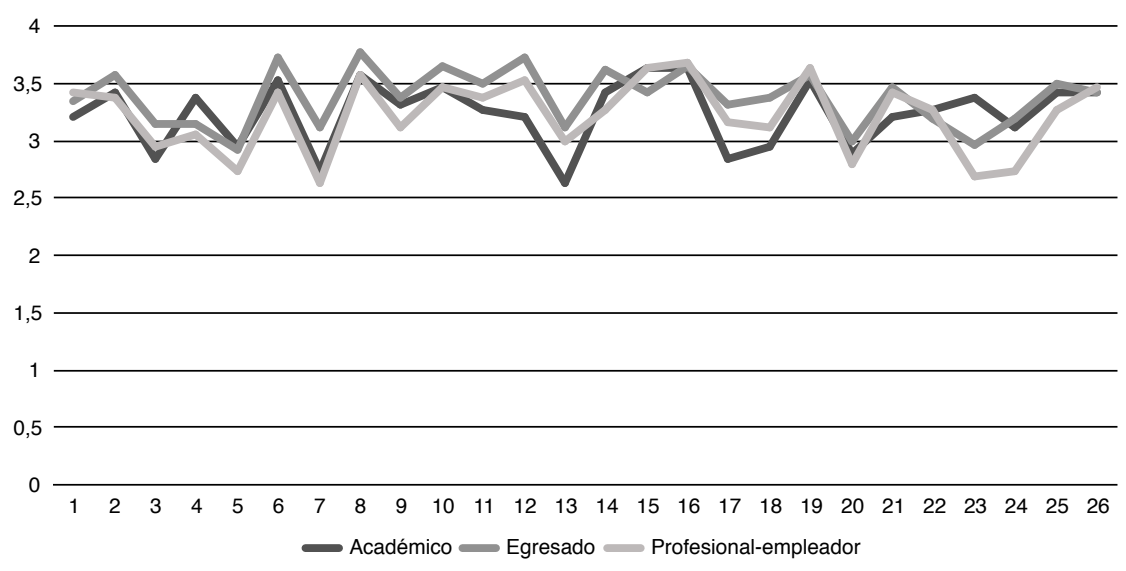

Fuente: Massaguer (2018). 
el soporte impreso y el digital y tener criterio para escogerlos según las necesidades del proyecto y la capacidad de realización de proyectos en nuevos formatos digitales de publicación.

En cuanto a las competencias transversales, se valora que tendrán más importancia las referidas a la competencia interpersonal capacidad de colaboración con diferentes agentes (CT9) y a la competencia sistémica capacidad para comprender e interpretar otros modos de vivir (CT13), así como la competencia instrumental uso fluido de diferentes idiomas (CT1). La competencia instrumental conocimientos en tareas contables (CT4) es la peor valorada.

Gráfico 4. Valoración de la importancia de las competencias transversales en los próximos 5 años, según implicados

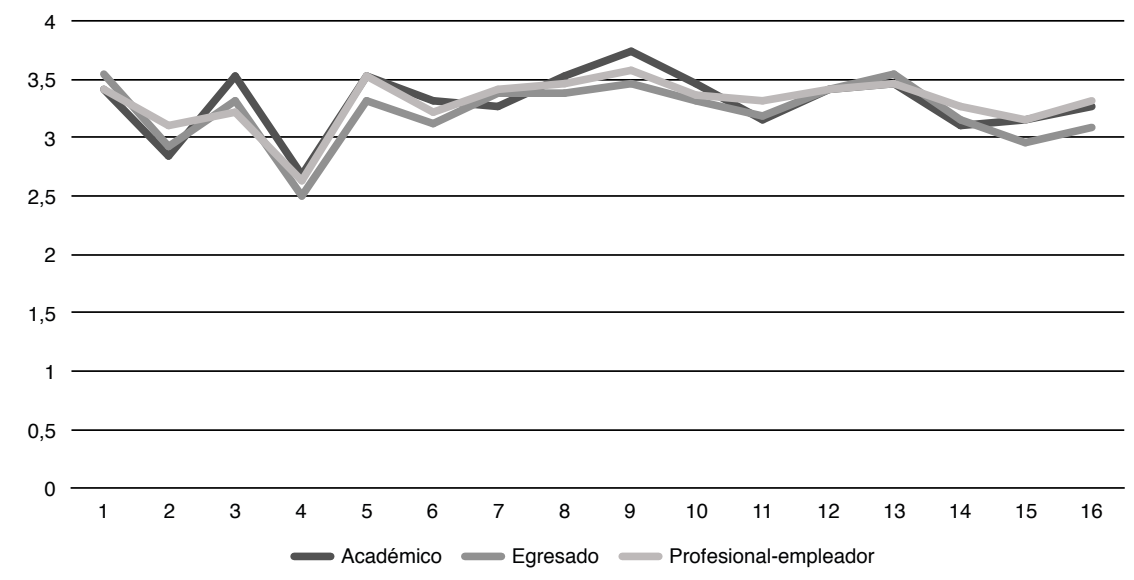

Fuente: Massaguer (2018).

$\mathrm{Al}$ analizar los resultados del focus group con graduados sobre el logro de las competencias en el ámbito académico y su ejercicio en el profesional, se ha llegado a las competencias que se consideran como logradas y aquellas que se ha percibido como carencia en su formación. Sintetizando, en el primer caso, en el grupo de competencias consideradas alcanzadas, entrarían las referidas al dominio de los criterios de usabilidad y tenerlos en cuenta a la hora de desarrollar proyectos de diseño (CE8), la capacidad para trabajar considerando la interactividad del usuario en los proyectos de diseño (CEG), el criterio para reconocer y escoger el contexto donde hay que actuar a través del diseño (CE19) o la capacidad para comprender e interpretar otros modos de vivir (CT13).

En el otro extremo encontramos aquellas competencias no logradas o con carencias en práctica en su ejercicio profesional, como son la capacidad para formalizar en el ámbito del diseño online (CE10), la capacidad para entender la convivencia y el diálogo entre el soporte impreso y el digital y tener criterio para escogerlos según las necesidades del proyecto (CE15), la capacidad de realización de 
proyectos en nuevos formatos digitales de publicación (CE12), la capacidad de colaboración con diferentes agentes (CT9) y el uso fluido de diferentes idiomas (CT1).

En el focus group los egresados propusieron mejoras competenciales en el actual plan docente, como puede ser que el conocimiento de los procesos, las tecnologias y los costes de fabricación y producción de los proyectos de diseño desarrollados sea un aspecto transversal en todo el grado, que se integre el conocimiento de programación en tecnologías digitales o que realizar los proyectos desde el inicio hasta el final sea una constante en todos los curriculos, dejando en minoritarios aquellos proyectos y ejercicios más parciales o que solamente tocan un elemento puntual del proceso de diseño.

El focus group con agentes académicos, que cierra la triangulación, ha permitido presentar los resultados del anterior focus grup con el fin de plantear los puntos que han creado tensión entre competencias académicas y profesionales y de conseguir llegar a propuestas que puedan ser aplicadas como mejora en el plan de estudios. De este encuentro han salido reflexiones y propuestas de mejora que posteriormente se han planteado en el cambio de plan docente del grado para el curso 2019-2020. Son ejemplo de ellas el aumento de estudiantes con un perfil no estrictamente de diseño gráfico, sino que tiende a la "hibridación" con la cultura del diseño, más enfocado a aquellos que tienden a la gestión empresarial, la gestión cultural, y también en los campos de la difusión, la crítica, la docencia y la investigación, más que a lo que entendíamos como perfil profesional de diseñador gráfico más formalizador, que se especializa en la tipografía, el diseño editorial, la identidad corporativa, etc.

\section{Discusión y conclusiones}

En línea con el objetivo de nuestra investigación, referido al análisis de la correspondencia entre las competencias del plan de estudios y la demanda que hace la profesión, hemos acotado previamente el perfil profesional de diseñador gráfico. En este sentido, la investigación ha pasado por concretar las competencias que requiere el ámbito profesional del diseño actualmente, partiendo de los referentes teóricos y normativos (perfil título ANECA) y desde la mirada de los actores implicados (Freire et al., 2013; González-Mardones, 2016).

Ello nos ha permitido confirmar tanto la importancia actual como la futura de las diferentes competencias del referido perfil (específicas y transversales), a la vez que también hemos podido verificar, a través de la metodología Delphi, el nivel de consenso existente entre los diferentes actores, tanto de la formación (académicos) como del empleo (profesionales empleadores y egresados), con lo que hemos constatado, pues, el nivel de correspondencia entre las competencias impartidas en la universidad y las requeridas en el ámbito profesional.

En síntesis, el diseñador gráfico trabaja en la actualidad con perfiles profesionales de diferentes ámbitos que no necesariamente tienen que ver directamente con el diseño (proveedores de diversos campos industriales, de la programación, la investigación, etc.); está atento a la innovación y a los cam- 
bios que se van produciendo, tanto en diseño gráfico como en sectores afines, y trabaja en equipos multidisciplinares y flexibles, desarrollándose por igual tanto en el ámbito analógico como en el digital. Tiene integrada una gran dosis de intuición, trabajo conceptual, creatividad y capacidad de resolución de problemas de diversa dificultad.

Las competencias académicas pueden ayudar a innovar en las competencias demandadas desde el ámbito profesional y al revés. Entendemos que es tan importante alcanzar los requisitos y las expectativas del campo profesional como la flexibilidad y la capacidad de innovación de la universidad para anticipar y responder a cuestiones y a requerimientos de los avances dentro de los diferentes campos profesionales (CEDEFOP, 2014, 2016, 2017; OECD, 2015; Pizarro-Barceló y Herrero-Montagud, 2016; Planas, 2014; RodríguezEsteban et al., 2019; Teichler, 2015).

La investigación nos ha permitido realizar un seguimiento continuo y valorativo del programa curricular del grado de Diseño, pero no desde una mirada endogámica, sino como puerta abierta a aportar mejoras y soluciones dentro de un campo de acción racional, no solo al ámbito formativo, sino también al profesional, teniendo siempre presente el contexto en el que se encuentran todos ellos (Rodríguez-Esteban et al., 2019; Somers et al., 2016). Asimismo, la presente investigación también quiere dar respuesta a la utilidad de la formación, a su transferencia en el lugar y en el escenario de trabajo, cumpliendo una función pedagógica como finalidad última (Tejada, 2012). Un camino de ida y vuelta que quiere beneficiar, finalmente, tanto al ámbito formativo como al profesional del diseño gráfico.

Una vez realizada la investigación y mirando al futuro para su aplicabilidad, es necesario valorar que la implantación de un sistema de seguimiento y de evaluación de competencias pide cambios, entre otros, también a las estructuras educativas (CEDEFOP, 2017; Freire et al., 2013). Las limitaciones de tiempo de trabajo, y en ocasiones también de formación, que tiene el profesorado necesitarían de, por ejemplo, la creación de unidades de apoyo para el diseño y el desarrollo de técnicas e instrumentos de evaluación de competencias o la conjugación de varias técnicas e instrumentos en la evaluación de competencias, tanto dentro como fuera del aula (Tejada y Ruiz, 2016; Vega, 2017).

Es decir, el camino está iniciado, pero habría una serie de recursos para poder aplicar, de manera regular y efectiva, el sistema diseñado. En nuestro caso, la metodología Delphi se constituye en un sensible y robusto dispositivo de diagnóstico para la actualización de las competencias del perfil académico, que está pensado para poderse aplicar de manera regular cada 5 años (Massaguer, 2018). Se trata de un "protocolo» que muestra los agentes informadores a quien recurrir, indicadores, tipo de acción y perfil que se analiza o se construye en cada una de sus etapas de aplicación. Quiere ser una herramienta que ayude a conocer las demandas del ámbito profesional del sector, así como actualizar las competencias del grado de Diseño, en este caso, pero extrapolable a otros títulos universitarios. Unas y otras se retroalimentarán. 


\section{Referencias bibliográficas}

Agencia Nacional de Evaluación de la Calidad y Acreditación (ANECA) (2004). Libro blanco: Titulos de grado en Bellas Artes/Diseño/Restauración. Recuperado de <http://www.aneca.es/var/media/150332/libroblanco_bellasartes_def.pdf>.

Agencia para la Calidad del Sistema Universitario de Cataluña (AQU) (2017). La inserció laboral dels graduats i graduades de les universitats catalanes. Barcelona: AQU.

Bartual, M.T. y Turmo, J. (2016). Educación superior y competencias para el empleo: El punto de vista de los empresarios. Revista Complutense de Educación, 27(3), 1211-1228. <https://doi.org/10.5209/rev_RCED.2016.v27.n3.47645>

Cabero, J. y Barroso, J. (2013). La utilización del juicio de experto para la evaluación de TIC: El coeficiente de competencia experta. Bordón, 65(2), 25-38. <https://doi.org/10.13042/brp.2013.65202>

Cabero, J. e Infante, A. (2014). Empleo del método Delphi y su empleo en la investigación en comunicación y educación. EDUTEC: Revista Electrónica de Tecnología Educativa, 48, 1-16. <https://doi.org/10.21556/edutec.2014.48.187>

Cabrera, N.; López López, M. y Portillo, M. (2016). Las competencias de los graduados y su evaluación desde la perspectiva de los empleadores. Estudios Pedagógicos, 3, 69-87.

CEDEFOP (2014). Desajuste de competencias: Más allá de lo que parece a simple vista. [Nota informativa.] Recuperado de <http://www.cedefop.europa.eu/files/9087_ es.pdf>.

- (2016). Escasez y exceso de cualificaciones y ocupaciones en Europa. [Nota informativa.] Recuperado de <http://www.cedefop.europa.eu/files/9115_es.pdf>.

- (2017). Anticipación de las competencias: Perspectivas futuras. [Nota informativa.] Recuperado de <http://www.cedefop.europa.eu/files/9124_es.pdf $>$.

CIDAC (2014). Encuesta de competencias profesionales 2014: ¿Qué buscan -y no encuentran - las empresas en los profesionales jóvenes? México: CIDAC.

Coller, X. (2000). Estudio de casos. Cuadernos Metodológicos. Madrid: CIS.

Consejo Económico y Social (CES) (2015). Informe competencias profesionales y empleabilidad. Madrid: Publicaciones CES.

Del Vecchio, F. (2014). Diálogos con diseñadores: Reflexiones sobre el deterioro profesional en el diseño gráfico, las soluciones habituales desde el diseño para enfrentarlo y otras propuestas desde la administración. Recuperado de <http://fernandodelvecchio. $\mathrm{com} /$ varios/DCD.pdf>.

Folch, A. (2016). Cómo será el futuro del diseño. Revista Gràffica, 1, 65-75.

Freire, M.J.; Teijeiro, M.M. y PAis, C. (2013). La adecuación entre las competencias adquiridas por los graduados y las requeridas por los empresarios. Revista de Educación, 362, 13-41.

<https://doi.org/10.4438/1988-592X-RE-2011-362-151>

García. V.; Aquino. S.; Guzmán, A. y Medina, A. (2012). El uso del método Delphi como estrategia para la valoración de indicadores de calidad en programas educativos a distancia. Revista Calidad en la Educación Superior, 3(1), 200-222.

González-Mardones, S. (2016). El diseño gráfico y sus profesionales: Retos y definiciones. [Tesis doctoral.] Barcelona: Universitat de Barcelona.

GonzÁlez, J. y WagenaAR, R. (2006). Tuning Educational Structures in Europe II: La contribución de las universidades al Proceso de Bolonia. Universidad de Deusto y 
Universidad de Groninga. Recuperado el 31 de marzo de 2016, de <http://www. deusto-publicaciones.es/ud/openaccess/tuning/pdfs_tuning/tuning12.pdf>.

Hernández-Sampieri, R.; Fernández-Collado, C. y Baptista-Lucio, P. (2014). Metodología de la Investigación. 6. ${ }^{\mathrm{a}}$ ed. México: Mc Graw-Hill.

Iriondo, I. y PÉrez-AmaraL, T. (2016). The effect of educational mismatch on wages in Europe. Journal of Policy Modeling, 38, 304-323. $<$ https://doi.org/10.1016/j.jpolmod.2015.12.008>

Kucel, A. y Vilalta-Bufí, M. (2013). Why do tertiary education graduates regret their study program?: A comparison between Spain and the Netherlands. Higher Education, 65(5), 565-579. $<$ https://doi.org/10.1007/s10734-012-9563-y>

Lluch, L.; Fernández-Ferrer, M.; Pons, L. y Cano, E. (2017). Competencias profesionales de los egresados universitarios: Estudio de casos en cuatro titulaciones. Qurriculum, 30, 49-64.

Massaguer, L. (2018). Construcció d'un dispositiu de diagnosi per determinar l'ajustament entre les competències acadèmiques i les professionals dels titulats del Grau de Disseny. [Tesis doctoral.] Departamento de Pedagogía Aplicada. Universidad Autónoma de Barcelona.

Michavila, F.; Martínez, J.M.; Martín-González, M.; García, F.J. y Cruz, J. (2016). Barómetro de empleabilidad y empleo de los universitarios en España, 2015. Madrid: Observatorio de Empleabilidad y Empleo Universitarios.

Morgado, A.; Sequeira, T.; Santos, M.; Ferreira-Lopes, A. y Reis, A. (2015). Measuring labour mismatch in Europe. Social Indicators Research, 129(1), 161-179. $<$ https://doi.org/10.1007/s11205-015-1097-0>

OECD (2015). Skills Strategy: Diagnostic report: Spain. Recuperado de <http://www. oecd.org/skills/nationalskillsstrategies/Diagnostic-report-Spain.pdf>.

Pizarro-Barceló, R. y Herrero-Montagud, M. (2016). Empleabilidad y déficit formativo de los egresados universitarios. Investigaciones de Economía de la Educación, 11(11), 835-850.

Planas, J. (2014). Adecuar la oferta de formación a la demanda de trabajo ¿es posible? México: ANUIES.

Rodríguez-Esteban, A.; Vidal, J. y Vieira, M.J. (2019). Un análisis de la empleabilidad de los universitarios en España a través del ajuste horizontal. Revista de Educación, 384, 229-254. $<$ https://doi.org/10.4438/1988-592X-RE-2019-384-411>

Sala, G.; Planas, J. y Van Rompaey, E. (2010). Especialidad de Formación, especialidad de empleo y resultados de inserción. ¿'Trabajan los jóvenes donde «deberían»? Sociología del Trabajo, 70, 87-10.

Somers, M.; Cabus, S.; Groot, W. y Maassen van den Brink, H. (2016). Horizontal Mismatch between Employment and the Field of Education: Evidence from a Systematic Literature Review. Tier Working Paper Series. Tier WP16/02.

Teichler, U. (2015). Changing perspectives: The professional relevance of higher education on the way towards the highly-educated society. European Journal of Education, 50(4), 461-477. $<$ https://doi.org/10.1111/ejed.12146>

TejadA, J. (2012). La alternancia de contextos para la adquisición de competencias profesionales en escenarios complementarios de educación superior: Marco y estrategia. Educación XX1, 15(2), 17-40.

$<$ https://doi.org/10.5944/educxx1.15.2.125> 
TEJADA, J. y Ruiz, C. (2016). Evaluación de competencias profesionales en Educación Superior: Retos e implicaciones. Educación XX1, 19(1), 17-38. $<$ https://doi.org/10.5944/educXX1.12175>

Vallor, R.R.; Yates, K.A. y Brody, M. (2016). Delphi Research Methodology Applied to Place-Based Watershed Education. Education Sciences, 6(42), 1-19. $<$ https://doi.org/10.3390/educsci6040042>

VEGA. E. (2017). Educación y diseño en tiempos de cambio. Experimenta Magazine, 76.

Verhaest, D.; Sellami, S. y VAN der Velden, R. (2017). Differences in horizontal and vertical mismatches across countries and fields of study. International Labour Review, 156(1), 1-23.

<https://doi.org/10.1111/j.1564-913X.2015.00031.x> 